\title{
Estrategias Digitales UTILIZADAS POR 7 EMPRESAS DURANTE EL COVID-I9: ESTUDIO DE CASOS
}

Macarena Suárez Blanco*

DOI: I0.5377/EYA.VIIII.IO5I3

Recibido: 09/07/2020 Aceptado:17/09/2020

\section{RESUMEN}

El objetivo de este artículo es mostrar cómo 7 empresarios que desempeñan su actividad en el departamento de Maldonado (Uruguay) han incursionado en el uso de herramientas digitales para poder hacer frente a la emergencia sanitaria causada por el COVID19, y poder seguir trabajando durante la misma y en la "nueva normalidad". Con este objetivo nos proponemos estudiar noticias locales y globales sobre el impacto de la emergencia sanitaria en Uruguay, bibliografía especializada sobre el uso de redes sociales y la capacidad de adaptación de las empresas frente a la incertidumbre para intentar comprender las formas de innovar y comunicarse con los clientes que han implementados diversos empresarios para no cerrar sus locales durante la emergencia sanitaria. En conclusión, en los testimonios de casos se han visualizado diversas formas de adaptación e innovación en los negocios para poder captar al cliente, comunicarse con ellos por los diversos medios y poder operar con todas las medidas sanitarias y de seguridad.

PALABRA CLAVES: redes sociales, comunicación organizacional, herramientas digitales, impacto en los negocios COVID-19.

JEL: M00, M39, M13

*Investigadora, Universidad Católica del Uruguay, correo electrónico: contadoramacarena@gmail.com Uruguay 


\title{
Use of Social Networks in 7 Companies During the Health Emergency by COVID-ig: Case Study
}

\author{
Macarena Suárez Blanco*
}

\section{DOI: 7/EYA.VIIII.IO5I3}

Recivied: 09/07/2020 Accepted:17/09/2020

\begin{abstract}
The objective of this article is to show how 7 entrepreneurs who carry out their activity in the department of Maldonado (Uruguay). They have dabbled in the use of digital tools to be able to face the health emergency caused by COVID19, and to be able to continue working during it and in the "new normal". With this objective we propose to study local and global news on the impact of the health emergency in Uruguay, specialized bibliography on the use of social networks and the ability of companies to adapt to uncertainty; to try to understand the ways to innovate and communicate with customers that various entrepreneurs have implemented so as not to close their premises during the health emergency. In conclusion, in the testimonies of cases, various forms of adaptation and innovation in business have been visualized in order to capture the client, communicate with them through various means and be able to operate with all health and safety measures.
\end{abstract}

KEYWORDS: social networks, organizational communication, impact on business COVID-19, digital tools.

JEL: M00, M39, M13

\footnotetext{
* Researcher, Catholic University of Uruguay, Email: contadoramacarena@gmail.com Uruguay
} 


\section{INTRODUCCIÓN}

El modelo comunicacional está cambiando se puede visualizar desde tres perspectivas principalmente a saber: desde la óptica del consumidor, quien se vuelve activo en las relaciones, desde el mercado que está fragmentado, y desde el auge de las tecnologías de la información (en adelante TIC's) (Kotler \& Amstrong, Marketing, 2012). A partir de la década de 1950 la comunicación se ha vuelto vital para la gestión corporativa en las organizaciones (Gutiérrez, 1999). Las redes sociales se han convertido en una de las primeras aliadas para las empresas en todo el mundo contar con comunicación directa con los clientes y poder hacer publicidad es fundamental para todo emprendimiento. Cada vez, los mercados están más complejos para operar y se hace imposible analizar todas las variables que inciden directa o indirectamente frente a determinada situación o acontecimiento, siendo, imposible predecir el futuro (Mintzberg, Quinn, \& Voyer, 1997).

Las empresas para poder sobrevivir en este mercado deben incluir en su ADN al marketing para ofrecer valor a sus clientes (Andrade, 2017), siendo, pilares dentro de éstas la innovación, vocación de servicio, atención al cliente y creatividad. En este sentido, se deben conocer los procesos para poder llevar a cabo aquellos que generen más valor para la organización (Player, 2003), durante épocas de incertidumbre es fundamental crear aprendizaje organizacional y cristalizarlo dentro de las organizaciones (Chiavenato, 2007). Este auge ha visto su incremento en las pequeñas y medianas empresas para afrontar la denominada "nueva normalidad", la crisis del coronavirus ha sido un punto de inflexión para que estas busquen adentrarse a las redes sociales (Puro Marketing, 2020). Las crisis para las empresas son constantes, por lo que, hay que buscar la forma de poder contar con habilidades para afrontarlas (Fener \& Cevik, 2015), éstas van a moldear las necesidades de los clientes tanto en el qué como en cuánto van a consumir (Schiffman, Lazar, \& Wisenblit, 2010) afectando en este sentido la planificación estratégica de la empresa (Johnson, Scholes, \& Whittington, 2006).
La intención de este artículo es mostrar las diversas posturas que tomaron 7 empresarios en el departamento de Maldonado (Uruguay) para con las herramientas digitales (redes sociales, publicidad, marketing telefónico, plataformas, páginas web, aplicaciones móviles, entre otros), como también, saber cuál de estas opciones han sido utilizadas para poder superar esta situación y los beneficios que aparejó para ellos. Lo que se expuso con anterioridad conlleva a la pregunta de investigación: ¿Qué herramientas digitales aplicaron los empresarios y por qué para poder afrontar la situación de emergencia sanitaria? Para poder brindar respuesta a esta pregunta se planteó la siguiente hipótesis "la emergencia sanitaria afectó de maneras diferentes a los empresarios, quienes buscaron maneras de obtener ingresos durante la pandemia en las herramientas digitales". El objetivo general en el que se enmarcó este artículo es "Conocer qué herramientas digitales utilizaron algunos empresarios en el departamento de Maldonado (Uruguay) y québeneficios conllevó para su negocio.

La primera sección de este artículo es un marco teórico sobre el impacto del coronavirus en Uruguay y las diversas medidas adoptadas, continúa con redes sociales y su implicancia en las empresas. La siguiente sección del artículo es acerca de la metodología utilizada, luego prosigue con los resultados que se obtuvieron en los 7 testimonios de casos de empresarios mientras que continúa con las reflexiones finales y las conclusiones del estudio.

\section{Marco concePtual}

\subsection{Impacto de la crisis del COVID19 en uruguay}

Fue el 13 de marzo de 2020 cuando se confirmaron los primeros 4 casos que el presidente de la República el Dr. Luis Lacalle Pou anuncia la emergencia sanitaria por el COVID19 en el país (Presidencia Uruguay, 2020) en esa misma rueda de prensa se anuncia el cierre parcial de fronteras se suspenden espectáculos públicos y se anuncia que no es obligatorio asistir a clases (El País Uruguay, 2020). Hacia el 14 de marzo de 2020 se suspenden actividades en museos y Biblioteca Nacional, y se habilita un teléfono de contacto con médicos gratuito para la población 
(Presidencia Uruguay, 2020). Al siguiente día también se suspenden actividades hípicas y salas de juego de azar (SINAE, 2020) siguió el cierre de puertos deportivos, restricción de vuelos comerciales, y mayor control -en todo sentido- (SINAE, 2020). Asimismo, a lo largo de los días se fueron tomando diversas medidas sanitarias, económicas, laborales, fiscales para poder afrontar la emergencia sanitaria. Las medidas fueron focalizadas a las necesidades que se constataban de la población.

En Uruguay la mayoría de las actividades continuaron funcionando, dado, que no se declaró cuarentena obligatoria. Sin embargo, se exhortó a la población a circular lo menos posible (Infobae, 2020), se apeló a la responsabilidad de los ciudadanos, y quien necesitará trabajar podía hacerlo, debido a que las empresas privadas, son fundamentales para la salida de la crisis (Vargas Llosa, 2020). En abril se creó un Comité Honorario de Asesores Científicos (El Observador, 2020) las medidas se están tomando con prudencia, asesorados por un comité de científicos contrastando con los demás países de Latinoamérica (BBC News, 2020). En mayo se comenzaron a reintegrar la actividad normal en diversas industrias, por medio de Protocolos de actuación e higiene, habilitados en páginas gubernamentales (Montevideo Portal, 2020). Los expertos expresan que se pueden retrasar los picos de casos con acciones rápidas por parte de los gobiernos (Herrero \& Belardo, 2020), con una correcta organización y medidas efectivas (Marti, 2020), la distancia social y el liderazgo político son fundamentales para poder hacer frente a la pandemia global (Riggirozzi, 2020). Muchas actividades continuaron operando, si bien, las escuelas y algunos restaurantes cerraron, la respuesta a la crisis vino dada por una actitud proactiva y por la cantidad de pruebas y rastreos que se hicieron a la población, ubicando a Uruguay en los primeros puestos de Latinoamérica (Margolis, 2020). Giovanni Escalante representante de la Organización Mundial y la Organización Panamericana de la Salud elogió la manera en que Uruguay está combatiendo al COVID19 y este éxito se debe a la existencia de un comando único y responsabilidad de la población (MonteCarlo, 2020).

\subsection{Marketing digital en las empresas}

Las empresas logran un mayor éxito cuando mezclan los mecanismos tradicionales y digitales, teniendo en cuenta el posicionamiento de estas (Porter, 2009). El Marketing digital se puede definir como las diversas estrategias que se realizan por medio de las plataformas informáticas para poder llegar a potenciales clientes (Selman, 2017) para lograr engagement de la comunidad estos, deben contener incentivos sociales, contenido que sea entretenido y logre que la comunidad pueda participar y a la vez dar feedback a estas publicaciones (Bradley \& McDonald, 2011), al día de hoy se producen cientos de interacciones entre la comunidad y las empresas, por medio de las redes de contacto (Awad, 2007).

Las redes sociales dan la posibilidad de realizar publicidad poder conversar y fidelizar a posibles clientes (Castello, Del Pino, \& Ramos , 2014) por medio de los valores corporativos y atributos de marca (Altamirano \& Silva, 2018) conociendo las necesidades del cliente (Schultz, Tannenbaum, \& Lauterborn, 1993). En este sentido las empresas se deben adaptar a trabajar por medio de actitudes y planteamientos nuevos (Mathison, Gándara, Primera, \& García, 2007) para ello deben utilizar los recursos que detenta la empresa de la manera más eficiente (Mazariegos, Águila, Pérez, \& Cruz, 2013) considerando aspectos tantos cuantitativos como cualitativos (González \& Arciniegas, 2016).

El Marketing Digital es un nuevo desafío para que las empresas puedan ganar visibilidad (Moschini, 2012) también pueden hacer una segmentación de clientes, promociones, y tener una comunicación más directa con éstos (Castillo, 2017). En este sentido, los consumidores pueden recibir las comunicaciones por diversos medios los cuales se ajusten a sus necesidades (Schultz, Tannenbaum, \& Lauterborn, 1993). Por ello, es vital segmentar eficazmente al público objetivo (Hoyos, Sabogal, \& Vargas, 2016). Para poder elaborar una estrategia de Marketing Digital se debe conocer a la audiencia objetiva, integrar y determinar los canales de comunicación para poder evaluarlos (Key \& Czaplewski, 2017). Los consumidores por estos medios de contacto tienen una participación activa, pudiendo comentar, publicar y recomendar a la 
empresa en cuestión (Kotler, Kartajaya, \& Setiawan, Marketing 4.0: Transforma tu estrategia para atraer al consumidor digital, 2017) por eso se visualiza que la gestión de la incertidumbre se vuelve un pilar relevante para muchas empresas frente a la comunicación corporativa (Xifra, 2020). Es fundamental que el empresario conozca la potencialidad de las redes sociales, dado que, de no ser así, no van a aprovechar todas las oportunidades que estas ofrecen (Valos, Habibi, Casidy, Driesener, \& Maplestone, 2016).

Las estrategias digitales se aplican para poder convertir en resultados las interacciones que presenta la organización con su comunidad (Selman, 2017) con pilares como divertir, informar y convertirse en la primera opción (Forbes, 2013). Por medio de un estudio realizado por Astor Warehouse se llegó a la conclusión que la crisis COVID-19 ha adelantado dos años la evolución del ecommerce (Aguilar, 2020).

\section{Metodología}

La metodología de este trabajo se basa en el estudio de siete testimonios de casos de empresarios y bibliografía especializada en la temática para abordar el tema del COVID-19 se estudiaron noticias locales y de organismos estatales. Se sustenta en la búsqueda de información recurriendo a varias fuentes de información (Yin, 2003); es cualitativa de índole documental, tipo descriptivo, de corte no experimental y transaccional.

Es de carácter cualitativo, ya que, se desean comprender percepciones y comportamientos frente a una misma amenaza (Strauss \& Corbin, 2002) en este sentido se busca conocer experiencias reales para que sea más sencilla la comprensión (Lincoln \& Guba, 1981), e indagar información para luego emitir un juicio (Ceballos \& Froylan, 2009).

La elección de la muestra para este informe fue de carácter no probabilístico, y elegida de forma intencional, los criterios que se eligieron para poder estudiarla son: primero que sean empresas, las cuales que han sido afectados por el COVID-19 y que hayan buscado una salida a la crisis por medio del uso de la tecnología de la información, segundo que antes de la emergencia sanitaria, no tuvieran una estrategia de comercio electrónico o marketing digital implementada, tercero se buscaron empresas de varios rubros para poder complementar el análisis.

Es descriptiva dado que intenta describir que ocurrió en las situaciones de diversos empresarios luego de que la pandemia los afectó y las formas que idearon para poder superar este momento. A su vez, tiene elementos explicativos debido a que intenta explicar un mismo fenómeno desde diversas ópticas y su forma de poder afrontarlo.

La búsqueda de la información para elaborar el marco teórico se sustentó en libros, artículos científicos publicados en EBSCO y Google académico. Mientras que la información asociada a las medidas del gobierno uruguayo frente al COVID-19 se sustentó en noticias, protocolos y artículos de fuentes del Gobierno (Parlamento, Sistema Nacional de Emergencias -SINAE-, Presidencia Gubernamental. Uy) por medio de noticias publicadas en diarios locales y globales.

\section{Discusión DE RESUlTAdos}

Los resultados obtenidos vienen dados por 7 testimonios de casos de empresarios en el Departamento de Maldonado, quienes, frente a la amenaza del coronavirus implementaron tecnologías de la información para poder seguir operando.

"En mi caso abrimos de forma presencial en el mes de mayo en si los casos no estaban aumentando muy fuerte y abrieron la mayor parte de las tiendas $y$ actividades por medio del cumplimiento de diversos protocolos y medidas de seguridad e higiene. Fue en un horario reducido, cómo no habia mucha gente en las calles, lo que haciamos era publicar en las redes sociales con varios hashtags y pagando publicidad en linea. En ese sentido, logramos captar la atención del público, mostrar nuestros productos que son de joyeria y que los mismos lo pudieran venir a comprar al local. También, hicimos envios, el problema de los envios es que a veces es un poco menor o más caro que el producto. Entonces, se le encarecía al cliente el regalo o accesorio que utilizaba. Por lo que, usamos mucho el mostrar por las redes y que vengan a buscar al local 
en el horario que teniamos. Incluso, enviamos fotos por vía telefónica y mensajes directos para decirles que producto teniamos disponible, a qué precio y que horario cumpliamos (Testimonio Nro. 1, empresario en el rubro de joyería, Maldonado, Uruguay, 29 de mayo de 2020)".

En este sentido se puede visualizar que el empresario de acuerdo con su testimonio se ha adentrado a las redes sociales para poder mejorar sus ventas y las ha utilizada como aliadas para poder comunicarse con sus clientes. Con este fin, se han vuelto fundamentales para este caso poder atraer a nuevos clientes. De esta manera la empresa ha ganado visibilidad para con estos durante las exhortaciones de circular lo menos posible. La forma de venta conforme con el testimonio ha cambiado totalmente, debido, a que antes se comunicaba de forma presencial y directa. Ahora está enviando fotos, audios por medio de las tecnologías de información de sus productos a los clientes potenciales de acuerdo con sus solicitudes y necesidades.

"Mi emprendimiento es una tienda de ropa, estuvimos cerrados, pero por las redes sociales logramos vender productos puntuales como uniformes o camperas. Generalmente, sacábamos fotos y la subiamos a las redes sociales o enviamos mediante marketing mails a nuestras clientes que teniamos sus contactos registrados. También, usamos publicidad online para poder llegara más clientes, especificando que solamente se vendia por agenda. Por este medio, logramos crear la necesidad en más de una de ellas, quienes posteriormente venian a comprar o a probar el producto que deseaban (por medio de citas y agenda), con todas las medidas de seguridad. También, en algunos casos nos giraban y les enviamos el producto que necesitaban en el talle y color solicitado. Ahora, que abrimos nos seguimos apoyando en estas herramientas y ya podemos recibir nuevos clientes, claramente, siguiendo todos los protocolos de seguridad $e$ higiene de las prendas y probadores. (Testimonio Nro. 2, empresario en el rubro de vestimenta y accesorios femeninos, Maldonado, Uruguay, 10 de junio de 2020)".

Al igual del caso anterior, este empresario expresa que ha podido combinar las redes sociales con la venta presencial agendando cita con los clientes, implementando las medidas de seguridad para el contacto con el mismo. En este caso, se visualiza que, por medio de la carga de fotos en diversas redes sociales como por medio de contacto directo por teléfono ha logrado captar la atención de sus clientes, y obtener ingresos durante los días que mantuvo su local cerrado por la emergencia sanitaria.

"Yo tengo un restaurante de venta de comida árabe, nunca cerré el local, pero si durante dos meses debi evitar la atención presencial. En esos meses se incentivó la venta mediante redes sociales y plataformas de delivery. Donde los clientes pedian el producto, lo preparábamos y venía el delivery a levantarlo para llevarlo a sus casas. El mayor tema son las altas comisiones que me cobran por el servicio, que encarece el producto. Y a los clientes le cobran un envio por el mismo. Ese es el mayor problema al que nos enfrentamos con esta modalidad. Sin embargo, tuvo un auge en el momento que se decretó la emergencia sanitaria y los clientes responsablemente cumplian con las exhortaciones del Gobierno. He pensado, en poder hacer una aplicación propia de mi restaurante para vender por ese medio. Al dia de hoy abrimos la puerta presencial, muchas personas nos encargan los productos vía telefónica y por medio de las redes sociales y luego le avisamos cuando está pronto y lo pasan a retirar por el local. (Testimonio Nro. 3, empresario en el rubro de gastronomía, Maldonado, Uruguay, 4 de junio de 2020)".

En tal sentido este empresario destaca en su testimonio de caso que ha cambiado su forma de operar y comunicarse con clientes por la emergencia sanitaria. Si bien, el mismo estuvo unos meses con su local cerrado al público, ha logrado comunicarse con sus clientes y potenciales clientes por medio de las diversas redes sociales y aplicaciones de delivery; ya que, los clientes no tienen una comunicación directa con ellos, sino, que lo hacen por intermedio de estas plataformas. Lo que genera que la comunicación sea más indirecta lo cual quiere variar por medio de una propia aplicación. Sin embargo, luego de la pandemia por medio de las redes sociales, ha logrado, retomar su contacto con los clientes, quienes acuden al local o llaman vía telefónica y lograr realizar el pedido para posteriormente levantarlo, sin pagar el envío o encarecer el producto por las comisiones. 
"En mi caso como contadora pública he dado a conocer mis servicios por medio de las redes sociales y aplicaciones de comunicación constante. Cuando se dieron a conocer los primeros casos no abri mi oficina de forma presencial por casi dos meses. Sin embargo, por estos medios estuve en contacto continuo con mis clientes, y pude acceder a nuevos contactos. La forma de comunicarme varió claramente, diversos medios, y horarios frente a la clásica reunión presencial y entrega de documentación, también reconozco que forma de comunicarme con los organismos también varió se amplió mucho más el tema de envios online expresando por ese medio lo necesario para poder lograr los objetivos y poder seguir con las operaciones. En el momento que se decretó la emergencia sanitaria muchos de ellos cerraron o limitaron la atención al público. Sin embargo, siguieron operando online. Al día de hoy, la mayoría ha abierto por medio de protocolos sanitarios $y$ por agenda. (Testimonio Nro. 4, empresario en el rubro de contabilidad, Maldonado, Uruguay, 27 de mayo de 2020)".

Frente a este caso se visualiza que al profesional le varió la forma de trabajo, dado, que el mismo acostumbra a trabajar por medio de reuniones presenciales y entrega de documentación en los diversos organismos públicos. Sin embargo, al cerrar la oficina por la emergencia sanitaria tuvo que actualizar el modelo de trabajo para poder comunicarse con los clientes y organismos públicos. Por lo que, en este sentido los medios y canales de comunicación variaron completamente cobrando una gran relevancia las aplicaciones móviles, correos electrónicos, como también redes sociales.

"Como Psicóloga estamos brindando un servicio a la comunidad, utilizamos las redes sociales de nuestra página para poder contactar con posibles clientes $y$ pacientes que necesiten una guia y acompañamiento para poder superar esta pandemia. En este sentido, las diversas plataformas para comunicarnos se han vuelta vitales, si bien, el contacto con el paciente no es el mismo que antes, no hay la misma cercanía, no hay intercambio de miradas, pero de todas maneras hemos logrado una conexión ba sido fundamental para poder estar en comunicación con ellos y guiarlos en este momento de incertidumbre y miedo. (Testimonio Nro. 5, empresario en el rubro psicología, Maldonado, Uruguay, 11 de junio de 2020)".
El caso del psicólogo es similar al contador un servicio que tenía una gran predominancia en reuniones presenciales varió frente a los cambios de la exhortación del gobierno de quedarse en las casas. Sin embargo, fue requerido cambiar la perspectiva y a la vez el enfoque por el que trabajar. Mediante ello, lograron variar su modelo de trabajo para poder hacerlo por medio de las plataformas de videoconferencia y con gran ayuda de las llamadas y mensajes telefónicos. En este caso, se expresa que el servicio cambio desde la perspectiva del profesional. Sin embargo, pudo prestarlo por estas herramientas, logrando su objetivo.

"En mi caso cuando comenzó la emergencia sanitaria, muchos de mis clientes no venian por la exhortación del Gobierno de no salir de casa. Uno de ellos, me consultó por la posibilidad de hacer el pedido vía telefónica, no lo habia hecho antes, dado que nunca lo necesité. Sin embargo, en este momento era una alternativa viable. Por lo que, le envié fotos y una lista de precios de los productos y me iba consultando aquellos que necesitaba para pasarle los precios, disponibilidad y marcas. Luego, el venía y lo levantaba o yo se lo llevaba a su casa. Frente, a los pagos lo haciamos mediante tarjeta o transferencia bancaria. En ese momento fue de gran ayuda para poder vender los productos y a la vez, lograr fidelizar a los clientes que querian evitar aglomeraciones y eran responsables frente a la situación de emergencia sanitaria. (Testimonio Nro. 6, empresario en el rubro almacén y venta de frutas $y$ verduras, Maldonado, Uruguay, 9 de junio de 2020)".

En el caso de este testimonio se visualiza que el local no cerró presencialmente dado que los productos que comercializa son de primera necesidad. Por lo que, va a continuar abierto. Sin embargo, debe adaptarse a que sus clientes intentan salir lo menos posible (debido a las exhortaciones del gobierno). Dado que debe actualizar su forma de comercializar sus productos. Un cliente le solicita si puede hacer el pedido vía telefónica y levantarlo, por más, que no sea la forma que acostumbra a comunicarse es una vía viable durante la emergencia sanitaria, dado; que es lo que el público le está demandando. La comunicación con este cliente también cambió, porque de ser un auto servicio para pasar a hacer un pedido vía telefónica. 
"Durante el proceso de emergencia de coronavirus; cómo counselors tuvimos una oferta de trabajo honorario desde el instituto de enseñanza donde nos recibimos, para por medio de una plataforma informática poder conectar con personas que necesitaban nuestros servicios, por todo lo ocurrido y la incertidumbre, pero no podian pagarlo. La idea era empezar a escuchar las necesidades de las personas que se habian inscripto, por medio de tres encuentros de 50 minutos mediante vídeo llamada. La plataforma unia la demanda y la oferta, también se ayudaron con redes sociales y canales de televisión. Primero estos encuentros nos causaron un impacto total como profesionales, ya que sentiamos del otro lado una fuerte desesperación por la situación que estaban atravesando y en nuestro sentido una escuch a dolorosa de las personas que nos llamaban. Al ser temas tan vulnerables, $y$ de supervivencia, necesitábamos ser más empáticos. Si bien, acá no hubo cuarentena, muchas industrias estuvieron cerradas y personas perdieron su empleo. Y esto me llevó a conocer realidades totalmente diferentes y que también estaban por fuera de mi núcleo familiar. Pero también, destacó en las comunicaciones la importancia de la escucha, cómo también, la búsqueda de solución de esas personas. Todos los sábados haciamos una reunión entre nosotros, los counselors que nos adherimos a prestar estos servicios; para poder ponernos de acuerdo y acompañarnos entre nosotros, cómo también; para poder conocer a quién delegar los casos que presentiamos más graves. (Testimonio Nro. 7, counselors, Maldonado, Uruguay, 13 de junio de 2020)".

Si bien este testimonio de casi se expresa que los servicios fueron sin costo para las personas, se puede dilucidar que los mismos fueron brindados por medio de una plataforma digital la cual gestionaba la demanda y la oferta del servicio, y por medio de ella se podían generar las reuniones. Por otro lado, frente a la comunicación se expresa la vulnerabilidad de las personas y su necesidad de ser escuchados y guiados. En ese sentido, es importante destacar la importancia de generar empatía y poder lograr que el mismo paciente pueda desplegar todo su problema para acompañarlo en la búsqueda de las posibles soluciones.

\subsection{Sintesis de los resultados obtenidos}

A modo de síntesis de los resultados obtenidos en los testimonios de casos de los empresarios se copilarán categorías de uso de las tecnologías de la información por los mismos en la siguiente tabla:

Tabla 1 sintesis de testimonios

\begin{tabular}{lccccccc}
\hline \multicolumn{1}{c}{ Síntesis } & $\mathrm{T} 1$ & $\mathrm{~T} 2$ & $\mathrm{~T} 3$ & $\mathrm{~T} 4$ & $\mathrm{~T} 5$ & $\mathrm{~T} 6$ & $\mathrm{~T} 7$ \\
\hline $\begin{array}{l}\text { Mayor uso de redes } \\
\text { sociales }\end{array}$ & $\mathrm{X}$ & $\mathrm{X}$ & $\mathrm{X}$ & $\mathrm{X}$ & $\mathrm{X}$ & & \\
$\begin{array}{l}\text { Uso de plataformas } \\
\text { especiales }\end{array}$ & & & $\mathrm{X}$ & & & & $\mathrm{X}$ \\
$\begin{array}{l}\text { Necesidad de usar vídeo } \\
\text { conferencias }\end{array}$ & & & & $\mathrm{X}$ & $\mathrm{X}$ & & $\mathrm{X}$ \\
$\begin{array}{l}\text { Envíos de email y por } \\
\text { contactos de clientes }\end{array}$ & $\mathrm{X}$ & $\mathrm{X}$ & $\mathrm{X}$ & $\mathrm{X}$ & & $\mathrm{X}$ & \\
$\begin{array}{l}\text { Uso de telefonía para } \\
\text { contacto y servicios }\end{array}$ & & & & & & & \\
\begin{tabular}{l} 
Pago de publicidad online \\
\hline
\end{tabular} & $\mathrm{X}$ & $\mathrm{X}$ & & & & & \\
\hline
\end{tabular}

Fuente: realización propia

\section{REFLEXIONES}

Dentro de los testimonios ellos destacan la relevancia que se ha tornado el uso de medio digitales para sus comercios lo que queda cuestionar es la aplicación que harán de estos de cara al futuro. Al igual que conocer cómo se ha generado las comunicaciones con los clientes, las variaciones de éstas como también la forma en que estos se dirigen a las empresas por más de un tipo de canal de comunicación. Los mismos han ahondado en diversas estrategias para enfrentar la pandemia y los perjuicios ocasionados por la misma muchos de ellos han optado por presentar una mayor presencia en las redes sociales generando contenido entretenido y de calidad para poder captar nuevos consumidores. Otros han elegido por la opción de plataformas especiales, o adaptar sus servicios a video conferencias para poder brindar el servicio, a pesar, de perder el contacto presencial. Por lo contrario, el envió de mails a contactos u uso de servicios de telefonía han sido elegido en varios de los testimonios. La publicidad online ha sido elegida en otras ocasiones, pero en estos casos no se ve un gran auge para todos ellos. Sin embargo, ha generado buenos resultados y una alternativa para captación de nuevos clientes a pesar de que sean más lejano, lo que se suma y va de la mano con la adaptación de los servicios para prestación a distancia. 
Es importante destacar que por medio de la exhortación del gobierno muchas de las industrias pudieron continuar operando como también las empresas, aunque la modalidad de servicio y propuesta de valor cambió en base a la responsabilidad de las personas y la menor circulación. Asimismo, es relevante cuestionar si consideran relevante poder invertir en estos medios y en publicidad para seguir ganando visibilidad, o si de lo contrario piensan que no es necesario y van seguía trabajando como hasta antes por medio de la presencialidad.

\section{Conclusiones}

A lo largo de este trabajo se intentaron contrastar diversos testimonios de casos de industrias variadas los cuales han buscado mantener activas sus emprendimientos por medio de la implementación de estrategias digitales como también de mantener el contacto con sus clientes y potenciales clientes durante las exhortaciones de circular lo menos posible se intentó también, abordar los problemas que enfrentaron las mismas durante este período.

Mediante estos testimonios se puede visualizar cómo estos empresarios vieron en las redes sociales una salida para la emergencia sanitaria sobre todo en el momento de mayor incertidumbre donde muchos de ellos cerraron sus locales a la atención al público debido a la exhortación de no salir de sus casas. En este sentido, si bien, no sustituyó la atención personal, si fue una medida que ayudó a amortiguar los gastos o poder tener ingresos para hacer frente a los gastos. Muchos continúan con el uso de estas herramientas digitales y comunicación por este medio con ello han logrado estar en contacto con potenciales clientes lo que genera un feedback directo como también, la complementación de los dos aspectos (presencialidad y virtualidad). Asimismo, logran obtener una mayor visibilidad a sus productos y servicios, y también atendiendo por medio de las redes sociales y telefonía logran generar un contacto antes con el cliente.

Esta situación azotó de diversa índole a las empresas, lo que conlleva, a la necesidad de reinventar las estrategias para poder amoldarse a la llamada "nueva normalidad", implementando todos los protocolos de acción como medidas sanitarias y de higiene.

La mayoría de ellos expresa que han tenido una reducción de ingresos a causa de la pandemia o han cerrado sus locales durante cierto período. Sin embargo, han podido continuar operando, manteniendo las fuentes de trabajo y contacto con los clientes.

\section{REFERENCIAS BIBLIOGRÁFICAS}

Altamirano, M., \& Silva, J. (2018). Capítulo 2. La importancia del personal branding para la competitividad laboral. En Innovación y competitividad de negocios, perspectivas para el desarrollo económico. Zona Centro Guadalajara, Jalisco: México: Ediciones.

Andrade, D. (2017). Estrategias de marketing digital en la promoción de Marca Ciudad. Rev. esc.adm.neg, 59-72.

Awad, E. M. (2007). Manual fundamental de comercio electrónico. Madrid: Anaya Multimedia.

BBC News. (29 de 05 de 2020). Coronavirus en Uruguay: la singular y exitosa estrategia del país para contener la pandemia sin cuarentena obligatoria. Obtenido de BBC News Mundo: https://www.bbc.com/mundo/ noticias-america-latina-528371935.

Bradley, A., \& McDonald, M. (2011). La Organización Social, Convertir en resultados las oportunidades de las redes sociales. Madrid: Editorial Profit.

Castello, A., Del Pino , C., \& Ramos , I. (2014). Twitter como canal de comunicación corporativa y publicitaria. Comunicación y Sociedad, 27(2), 21-54.

Castillo, R. (2017). 24 ventajas de tener un sitio Web para cualquier negocio. Obtenido de MundoVirtual. : https://www.mundovirtual.biz/24-ventajas-de-tenerun-sitio-web-para-cualquier-negocio/

Ceballos, H., \& Froylan, A. (2009). El informe de investigación con estudio de casos. Magis. Revista Internacional de Investigación en Educación, 1(2), 413423.

Chiavenato, I. (2007). Administración de recursos humanos El capital humano de las organizaciones. México: McGRAWHILL/INTERAMERICANA EDITORES, S.A. DE C.V. 
El Observador. (18 de 04 de 2020). La "nueva normalidad" y su funcionamiento con "pasos cortos". Obtenido de El Observador: https://www.elobservador.com.uy/nota/ la-nueva-normalidad-y-su-funcionamiento-con-pasoscortos--20204172330

El País Uruguay. (14 de 03 de 2020). Coronavirus en Uruguay: cierre parcial de fronteras y suspenden espectáculos públicos. Obtenido de El País: https:// www.elpais.com.uy/informacion/politica/gobiernodecidio-cierre-relativo-fronteras-confirmacioncoronavirus.html

Fener, T., \& Cevik, T. (2015). Leadership in Crisis Management: Separation of Leadership and Executive Concepts. Procedia Economics and Finance , 26, $695-$ 701.

González, O., \& Arciniegas, J. (2016). Sistemas de gestión de calidad. . Ecoe Ediciones.

Gutiérrez, M. (1999). Teoría matemática de la comunicación y teoría semántica de la información. Teorema Vol. XVIII/2, p. 77-100 https://dialnet. unirioja.es/descarga/articulo/4254150.pdf .

Herrero, M., \& Belardo, M. (05 de 04 de 2020). Covid-19 y la necesidad de rediscutir nuestros sistemas de salud. Obtenido de El Pais: https://www.elpaisdigital.com. ar/contenido/covid-19-y-la-necesidad-de-rediscutirlos-sistemas-\%20de-salud/26294

Hoyos, A., Sabogal, M., \& Vargas, C. (2016). Estimación de las relaciones entre la inversión en medios digitales y las variables financieras de la empresa: una aproximación para ColombiaBogotá. Revista Escuela de Administración de Negocios (EAN), 12-25.

Infobae. (18 de 05 de 2020). Cómo logró Uruguay controlar el coronavirus sin cuarentena obligatoria. Obtenido de Infobae: https://www.infobae.com/america/americalatina/2020/05/18/como-logro-uruguay-controlar-elcoronavirus-sin-cuarentena-obligatoria/

Johnson, G., Scholes, K., \& Whittington, R. (2006). DIRECCIÓN ESTRATÉGICA. SÉPTIMA EDICIÓN. Madrid: Editorial Pearson.
Key, T., \& Czaplewski, A. (2017). Upstream social marketing strategy: An integrated marketing communications approach. Business Horizons, 9.

Kotler, P., \& Amstrong, G. (2012). Marketing (Vol. Decimocuarta edición.). México, México, México: Pearson educación.

Kotler, P., Kartajaya, H., \& Setiawan, I. (2017). Marketing 4.0: Transforma tu estrategia para atraer al consumidor digital. United States of America: Wiley.

Lincoln, Y., \& Guba, E. (1981). Effective evaluation. San Francisco, California:: Jossey-Bass.

Margolis, M. (29 de 05 de 2020). Why Is Uruguay Beating Latin America's Coronavirus Curse? Obtenido de Bloomberg Opinion: Politics \& Policy: https:// www.bloomberg.com/opinion/articles/2020-05-29/ uruguay-is-beating-latin-america-s-coronaviruscurse

Marti, W. (05 de 06 de 2020). Abseits der grossen Schlagzeilen leistet Uruguay bemerkenswerte Arbeit bei der CoronaBekämpfung. Obtenido de NZZ: https://www.nzz. $\mathrm{ch} /$ international/coronavirus-uruguay-macht-einenbemerkenswerten-job-ld.1559464

Mathison, L., Gándara, J., Primera, C., \& García, L. (2007). Innovación: factor clave para lograr ventajas competitivas. Revista Negotium, 65-83.

Mazariegos, A., Águila, J., Pérez, M., \& Cruz, R. (2013). El control interno de una productora de café certificado.

Mintzberg, H., Quinn, J., \& Voyer, J. (1997). El proceso estratégico: conceptos, contextos y casos. Edición Breve. Mexico: Pearson Educación Mexico.

Moschini, S. (2012). Claves del Marketing Digital. Barcelona: La Vanguardia Ediciones.

MonteCarlo. (12 de 06 de 2020). Escalante elogió el combate de Uruguay al coronavirus COVID-19. Obtenido de MonteCarlo: https://www.radiomontecarlo. com.uy/2020/06/12/nacionales/escalante-elogio-el combate-de-uruguay-al-coronavirus-covid-19/ 
Montevideo Portal. (29 de 04 de 2020). PASITO A PASITO: Gobierno planea nuevo paso hacia la "nueva normalidad" con el regreso del sector público. Obtenido de Montevideo Portal: https://www.montevideo.com. uy/Noticias/Gobierno-planea-nuevo-paso-hacia-lanueva-normalidad-con-el-regreso-del-sector-publicouc751471

Player, S. (2003). Why some organizations go "Beyond Budgeting". The Journal of Corporate Accounting and Finance, pp.3-9.

Porter, M. (2009). Ser competitivo. Madrid: Ediciones Deusto.

Presidencia Uruguay. (13 de 03 de 2020). Gobierno declaró emergencia sanitaria por coronavirus y anunció las primeras medidas. Obtenido de Presidencia Uruguay: https://www.presidencia.gub.uy/comunicacion/ comunicacionnoticias/lacalle-medidas-coronavirusconferencia

Presidencia Uruguay. (14 de 03 de 2020). Presidencia Uruguay. Obtenido de Gobierno definió suspensión de clases en centros educativos públicos y privados de todo el país por próximos 14 días: https://www.presidencia. gub.uy/comunicacion/comunicacionnoticias/ conferencia-durazno-suspension-clases-coronavirusmsp

Puro Marketing. (10 de 05 de 2020). Ocho de cada 10 pymes consideran imprescindible para su negocio mantener la actividad online en la "nueva normalidad". Obtenido de Puro Marketing: https://www.puromarketing. com/14/33846/ocho-cada-pymes-consideranimprescindible-para-negocio-mantener-actividadonline-nueva.html

Riggirozzi, P. (30 de 3 de 2020). CORONAVIRUS Y EL DESAFÍO PARA LA GOBERNANZA REGIONAL EN AMÉRICA LATINA. Fundación Carolina, marzo 2020, págs. 1-13.

Schiffman, L., Lazar, L., \& Wisenblit, J. (2010). Comportamiento del consumidor. DÉCIMA EDICIÓN. México: Editorial Pearson.

Schultz, D., Tannenbaum, S., \& Lauterborn, R. (1993). Comunicaciones de marketing integradas. Madrid:
Ediciones Granica S.A.

Selman, H. (2017). Maketing Digital. Índigo Estudio Ibukko.

SINAE. (15 de 03 de 2020). Cierre de salas de juegos de azar administradas por la Dirección General de Casinos y suspensión de competencia hipica. Obtenido de SINAE: https://www.gub.uy/ministerio-economia-finanzas/ comunicacion/noticias/cierre-salas-juegos-azaradministradas-direccion-general-casinos-suspension

SINAE. (16 de 03 de 2020). INFORME DE SITUACIÓN EN RELACIÓN AL PLAN NACIONAL CORONAVIRUS. Obtenido de SINAE: https://www.gub.uy/sistemanacional-emergencias/sites/sistema-nacionalemergencias/files/2020-03/informe\%20 de $\% 20$ situación $\% 20$ coronavirus $\% 20 \% 2816 \% 20 \mathrm{de} \% 20$ marzo\%293.pdf

Strauss, A., \& Corbin, J. (2002). Strauss, A. y Corbin, J. (2002). Bases de la investigación cualitativa. Técnicas y pro-cedimientos para desarrollar la teoría fundamentada. Medellín: Editorial Universidad de Antioquia.

Valos, M., Habibi, F., Casidy, R., Driesener, C., \& Maplestone, V. (2016). Exploring the integration of social media within integrated marketing communication frameworks: perspectives of services marketers. Marketing intelligence and planning, 1-33.

Vargas Llosa, M. (07 de 06 de 2020). https:/www. latercera.com/la-tercera-domingo/noticia/columnade-mario-vargas-1losa-el-ejemplo-uruguayo/ A2BMPUGHEFGT5G5B2QFYS6V27M/. Obtenido de La Tercera: https://www.latercera.com/la-tercera-domingo/ noticia/columna-de-mario-vargas-llosa-el-ejemplo-uruguayo/ A2BMPUGHEFGT5G5B2QFYS6V27M/

Xifra, J. (2020). Comunicación corporativa, relaciones públicas y gestión del riesgo reputacional en tiempos del Covid-19. El profesional de la información, 29(2), 1-19.

Yin, R. (2003). Case study research: Design and methods (3rd ed.). . Thousand Oaks, CA: Sage 


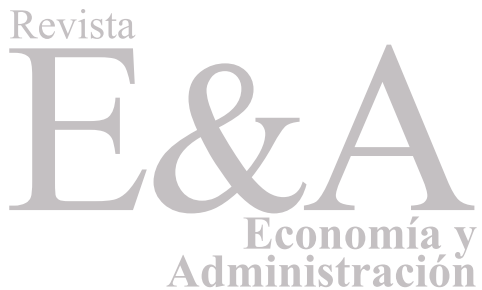

